\title{
The Effect of One Anastomosis Gastric Bypass on Branched-Chain Fatty Acid and Branched-Chain Amino Acid Metabolism in Subjects with Morbid Obesity
}

\author{
Alicja Pakiet ${ }^{1}$ - Maciej Wilczynski ${ }^{2}$ - Olga Rostkowska ${ }^{2}$. Justyna Korczynska ${ }^{3}$. Patrycja Jabłonska ${ }^{4}$ - Lukasz Kaska $^{2}$. \\ Monika Proczko-Stepaniak ${ }^{2}$ - Ewa Sobczak ${ }^{5,6} \cdot$ Piotr Stepnowski $^{1} \cdot$ Faidon Magkos $^{7} \cdot$ Tomasz Sledzinski $^{3}$ (D) \\ Adriana Mika ${ }^{1,3}$
}

Published online: 22 August 2019

(C) The Author(s) 2019

\begin{abstract}
Background Subjects with morbid obesity have low levels of serum branched-chain fatty acids (BCFAs), which correlate inversely with insulin resistance, hypertriglyceridemia, and inflammation. Recent evidence suggests BCFAs are produced during branched-chain amino acid (BCAA) catabolism in human adipose tissue. Elevated concentrations of BCAAs are associated with insulin resistance.

Objectives In this single-center study, we evaluated the effect of one anastomosis gastric bypass (OAGB) on circulating BCFA and BCAA. Moreover, we determined the expression of genes involved in BCAA catabolism in adipose tissue of patients with obesity and lean controls.

Methods Fasting levels of BCFAs and BCAAs were determined by gas and liquid chromatography, respectively, coupled with mass spectrometry, in 50 patients with morbid obesity before and 6-9 months after surgery, and in 32 lean controls. Visceral and subcutaneous adipose tissue (VAT and SAT, respectively) biopsies were collected at baseline to determine mRNA levels for enzymes involved in BCAA catabolism.

Results Before surgery, patients with obesity had lower BCFAs and greater BCAAs than control subjects. OAGB increased BCFA and decreased BCAA levels. Insulin resistance (assessed by HOMA) correlated inversely with BCFAs and positively with BCAAs. Expression of genes involved in BCAA catabolism in VAT (but not SAT) was lower in patients with obesity than in lean controls.
\end{abstract}

Electronic supplementary material The online version of this article (https://doi.org/10.1007/s11695-019-04157-z) contains supplementary material, which is available to authorized users.

Tomasz Sledzinski tsledz@gumed.edu.pl

Alicja Pakiet

alicjapakiet@gmail.com

Maciej Wilczynski

wilczynskimj@gmail.com

Olga Rostkowska

olgaa.rostkowska@gmail.com

Justyna Korczynska

justyna.korczynska@gumed.edu.pl

Patrycja Jabłonska

patrycja.jablonska@gumed.edu.pl

\author{
Lukasz Kaska \\ lukasz.kaska@wp.pl \\ Monika Proczko-Stepaniak \\ mproczko@gumed.edu.pl \\ Ewa Sobczak \\ ewa.sobczak@gumed.edu.pl \\ Piotr Stepnowski \\ piotr.stepnowski@ug.edu.pl \\ Faidon Magkos \\ fma@nexs.ku.dk \\ Adriana Mika \\ adrianamika@o2.pl
}

Extended author information available on the last page of the article 
Conclusions OAGB-induced weight loss increases circulating BCFAs and decreases circulating BCAAs in patients with morbid obesity, perhaps by altering BCAA catabolism in VAT. We speculate that this shift may be related to the improvement in insulin sensitivity after surgery.

Keywords Branched-chain amino acids $\cdot$ Branched-chain fatty acids $\cdot$ Bariatric surgery $\cdot$ Morbid obesity $\cdot$ Insulin resistance

\section{Introduction}

Bariatric surgery is a treatment option for morbid obesity and is more effective than diet and pharmacotherapy $[1,2]$. Bariatric surgery brings about significant alterations in lipid metabolism, but the exact nature of these changes depends on the surgical procedure [3]. One anastomosis gastric bypass (OAGB) is a promising type of bariatric surgery that improves metabolic parameters (reduction in serum concentrations of triglycerides, total and LDL cholesterol, and C-reactive protein; and increase in HDL cholesterol concentration) [4], causes remission of type 2 diabetes mellitus, and is associated with relatively low morbidity $[5,6]$. However, some studies found higher incidence of diarrhea, steatorrhea, and nutritional adverse events after OAGB than after RYGB [7]. Reduced food intake after surgery may result in deficiencies of some important nutrients [8], such as bioactive lipids which, although present in small amounts in the diet, are vital for many physiological functions. On the other hand, bariatric surgery may lead to an increase in serum concentrations of some other lipid classes, e.g., bile acids [3, 4, 9].

Branched-chain fatty acids (BCFAs) and odd-chain fatty acids (OCFAs), both present in small amounts in human blood, possess antibiotic, anticancer, and immunosuppressive properties [10-12]. Similar to unsaturated fatty acids, BCFAs can increase cell membrane fluidity, but are less prone to oxidation than the former [13]. OCFAs have antioxidant properties, acting as hydroxyl radical scavengers, and increased dietary intake of OCFA was shown to be associated with a decrease in serum cholesterol. Accordingly, consumption of BCFAs and OCFAs may reduce the risk of cardiovascular disease $[10,14]$ and metabolic disorders [15]. These fatty acids originate from ruminant fat and milk [16], but recent evidence suggests that both BCFAs and OCFAs can also be synthesized in mammalian adipocytes from branched-chain amino acids (BCAAs) [17-19]. Wallace et al. [19] demonstrated that BCFAs were synthesized de novo in adipose tissue from BCAAs catabolized in mitochondria, and then exported by carnitine acetyltransferase to the cytosol, where they were elongated by fatty acid synthase. In a previous study, we identified several BCFAs and OCFAs in human serum, and demonstrated that patients with morbid obesity without any dietary or surgical treatment had significantly lower levels of isoBCFAs than lean controls, but similar OCFA levels [16]. We also found inverse correlations between circulating isoBCFAs and C-reactive protein (CRP, an inflammatory marker), hypertriglyceridemia, and insulin concentration in patients with morbid obesity [16]. In contrast to BCFAs and OCFAs, elevated concentrations of BCAAs are associated with obesity and its metabolic complications, such as insulin resistance [17], and many authors have reported alterations in BCAA metabolism in insulin-resistant and obese states [17, 20,21].

Accordingly, the aim of this study was to evaluate if OAGB-induced weight loss affects circulating levels of bioactive fatty acids, including BCFAs and OCFAs. We also determined BCAAs as well as the expression of genes involved in BCAA catabolism in visceral and subcutaneous adipose tissues.

\section{Materials and Methods}

\section{Patients}

This single-center study included a group of 50 patients (mean age $48.6 \pm 10.5$ years; 42 females, 8 males) operated for morbid obesity at the Department of General, Endocrine and Transplant Surgery (Medical University of Gdańsk, Poland) between 2016 and 2018. There were 25 patients with type 2 diabetes (T2DM) and 25 without. The inclusion criteria for OAGB laid out by the International Federation for the Surgery of Obesity (IFSO), International Federation for the Surgery of Obesity-European Chapter (IFSO-EC), and European Association for the Study of Obesity (EASO) were applied [22].

The exclusion criteria for OAGB were as follows: (i) absence of a period of identifiable medical management; (ii) inability to participate in prolonged medical follow-up; (iii) non-stabilized psychotic disorders, severe depression, personality, and eating disorders unless specifically advised by a psychiatrist experienced in obesity; (iv) alcohol abuse or drug dependencies; (v) diseases threatening life in the short term; (vi) inability to self-care and lack of long-term family or social support. Blood samples were obtained from all patients before, and then 6-9 months after surgery.

The control group consisted of 32 lean individuals (mean age $52 \pm 12$ years; 21 females, 9 males) subjected to laparoscopic cholecystectomy at the Department of General, Endocrine and Transplant Surgery, Medical University of Gdansk, and the Department of General Surgery, Pomeranian Hospital Sp. z o.o., in Gdynia. Criteria for 
including patients in the control group included indications for surgical treatment in planned mode for non-cancerous reasons. Exclusion criteria were (i) obesity, (ii) diabetes, (iii) metabolic syndrome, (iv) steroid therapy, (v) chronic use of non-steroidal anti-inflammatory drugs, and (vi) acute inflammation. Blood samples from lean patients who would undergo cholecystectomy were obtained before surgery. The blood samples were collected in the morning both from patients and lean controls.

The study was performed in agreement with the principles of the Declaration of Helsinki of the World Medical Association. The study protocol was approved by the Local Bioethics Committee at the Medical University of Gdansk (decision no. NKBBN/493/2016) and written informed consent was obtained from all participants.

\section{Surgical Technique and Adipose Tissue Biopsies}

The one anastomosis gastric bypass (OAGB) procedure was performed laparoscopically in all patients with obesity. The length of the intestinal limb excluded from mixed food and biliopancreatic content in OAGB was estimated intraoperatively to be $180-250 \mathrm{~cm}$, depending on patient's BMI, age, and diabetes status, following the rule by Garcia-Caballero et al. [23]. The length of the left common limb was estimated to be $350-400 \mathrm{~cm}$. The measurement of intestinal length was based on the not-strained organ manipulation and the $10-\mathrm{cm}$ marker fixed to the grasping instrument that was used for calibration. Those procedures were performed laparoscopically. After a leakage test was conducted, in order to exclude potential leaks from the anastomosis, visceral adipose tissue (VAT) samples were collected. Upon closing of the wound, samples of subcutaneous adipose tissue (SAT) were also collected. All adipose tissue samples were immediately frozen in liquid nitrogen and stored at $-80^{\circ} \mathrm{C}$. Adipose tissue samples were obtained from the same anatomical locations from the group of lean controls during cholecystectomy. Adipose tissue in both groups has been collected intraoperatively, at the end of the procedure, always from the greater omentum and from the subcutaneous tissue at closing the central trocar.

\section{Anthropometric and Metabolic Data}

Anthropometric and laboratory parameters were determined at baseline (before surgery) in patients with morbid obesity and in lean subjects, and again 6-9 months after surgery only in subjects with morbid obesity. All blood samples were collected after an overnight fast. Serum was obtained by centrifugation and stored at $-80^{\circ} \mathrm{C}$. Routine laboratory parameters were determined at the Central Clinical Laboratory, Medical University of Gdansk. Anthropometric and metabolic data of patients with morbid obesity and lean controls are presented in Table 1.

\section{Chromatographic and Mass Spectrometry Analysis}

Fatty acid levels were assayed as described previously [16] by using gas chromatography-mass spectrometry (GC-MS) in total lipids extracted from tissues and serum using the method described by Folch et al. [24]. Serum amino acid concentrations were assayed by high-performance liquid chromatography-tandem mass spectrometry (LC-MS) according to Olkowicz et al. [25]. The details of these analytical techniques are provided in Supplementary File 1.

\section{Real-time PCR Analysis of mRNA Levels in Adipose Tissue}

Total RNA was extracted from frozen VAT and SAT samples with Qiagen RNeasy Lipid Tissue Mini Kit. The yield and quality were determined for each sample by automated gel electrophoresis (Experion, Bio-Rad). cDNA was synthesized from $0.5 \mu \mathrm{g}$ of the total RNA using RevertAid ${ }^{\mathrm{TM}}$ First Strand cDNA Synthesis Kit (Thermo Fisher Scientific). Each RNA sample was treated with RNase-free DNase I before the cDNA synthesis. mRNA levels were analyzed with real-time PCR using CFX Connect Real-Time System (Bio-Rad). A combination of $\beta$-actin and cyclophilin genes was used as reference standards. Relative levels of mRNA for the branched-chain $\alpha$ ketoacid dehydrogenase (BCKDH) complex and branchedchain aminotransferase (BCAT) were calculated according to Piehler et al. [26]. The following primer sequences were used: F: GATGACAAGCCCCAGTTCCCA, R': TGGGGTTG ATGATCTGGCCTT for BCKDHA; F': GCGGCAGG TGGCTCATTTTACT, R: CAGTAGGATCTTTGGCCAAT GAGTTAT for BCKDHB; F: GGTCCCATATTCAA CATCTGCTAGTCT, R: TCCCATCTTGCAGTCCCCAGT for BCAT1, and F: TTACGCGCCGCACGGATCAT, R: GGTCGGTAAATGTCTTCCCAAAC for BCAT2. Amplification of specific transcripts was confirmed based on melting curve profiles and agarose gel electrophoresis of the amplification products.

\section{Statistical Analysis}

The statistical significance of differences between values before and after surgery in subjects with morbid obesity was determined with paired $t$ tests, whereas differences between subjects with morbid obesity and lean controls were evaluated by independent $t$ tests. The differences were considered statistically significant at $p<0.05$. The relationship between variables of interest was determined with linear regression analysis and multiple linear regression analysis. All statistical analyses were carried out with SigmaPlot version 11.0 (Systat Software Inc., San Jose, CA). 
Table 1 Metabolic characteristics of patients with morbid obesity before and after surgery compared with lean controls. Values are mean $\pm \mathrm{SD}$. $L C$ lean controls, $O A G B$ one anastomosis gastric bypass, $B M I$ body mass index, $T G$ triglycerides, $H D L$ high-density lipoprotein cholesterol,
$L D L$ low-density lipoprotein cholesterol, $T C$ total cholesterol, $C R P$ Creactive protein, HOMA homeostatic model assessment of insulin resistance

Lean controls (LC) Pre-OAGB $\quad 6-9 \mathrm{~m}$ post-OAGB $\quad p$ (pre-OAGB vs LC) $\quad p$ (pre- vs post-OAGB) $\quad p$ (post-OAGB vs LC)

\begin{tabular}{|c|c|c|c|c|c|c|}
\hline BMI $\left(\mathrm{kg} / \mathrm{m}^{2}\right)$ & $24.3 \pm 2.95$ & $38.5 \pm 4.25$ & $29.6 \pm 3.73$ & $<0.001$ & $<0.001$ & $<0.001$ \\
\hline Age (years) & $52 \pm 12$ & $48.6 \pm 10.5$ & - & 0.456 & - & - \\
\hline $\operatorname{HbA1C}(\%)$ & - & $5.76 \pm 0.89$ & $5.22 \pm 0.46$ & - & 0.002 & - \\
\hline TG (mg/dl) & $104 \pm 43.5$ & $113 \pm 37.3$ & $87.8 \pm 26.7$ & 0.775 & 0.006 & 0.149 \\
\hline HDL (mg/dl) & $57.4 \pm 22.1$ & $50.7 \pm 9.52$ & $51.6 \pm 11.67$ & 0.066 & 0.590 & 0.109 \\
\hline LDL (mg/dl) & $97.6 \pm 37.4$ & $114 \pm 33.8$ & $88.3 \pm 25.3$ & 0.093 & 0.027 & 0.066 \\
\hline $\mathrm{TC}(\mathrm{mg} / \mathrm{dl})$ & $171 \pm 48.3$ & $201 \pm 39.8$ & $181 \pm 49.1$ & 0.002 & 0.015 & 0.189 \\
\hline CRP (mg/l) & $1.60 \pm 1.20$ & $1.65 \pm 0.53$ & $1.02 \pm 0.55$ & 0.904 & 0.010 & 0.170 \\
\hline Albumin (g/l) & $40.3 \pm 2.94$ & $37.4 \pm 7.60$ & $37.2 \pm 2.47$ & 0.018 & 0.832 & $<0.001$ \\
\hline Creatinine (mg/dl) & $0.85 \pm 0.19$ & $0.79 \pm 0.22$ & $0.71 \pm 0.17$ & 0.287 & $<0.001$ & 0.002 \\
\hline Glucose (mg/dl) & $96.8 \pm 2.32$ & $110 \pm 31.6$ & $91.5 \pm 10.9$ & 0.057 & $<0.001$ & 0.208 \\
\hline Insulin $(\mu \mathrm{U} / \mathrm{ml})$ & $9.58 \pm 4.79$ & $14.9 \pm 7.69$ & $7.70 \pm 6.27$ & 0.002 & $<0.001$ & 0.204 \\
\hline HOMA-IR & $2.48 \pm 2.14$ & $4.34 \pm 2.96$ & $2.01 \pm 1.89$ & 0.008 & $<0.001$ & 0.384 \\
\hline
\end{tabular}

\section{Results}

\section{The Effect of OAGB on Serum FA Composition}

Compared with lean controls, patients with morbid obesity had significantly lower levels of total BCFAs before surgery; however, OAGB-induced weight loss ( $23 \%$ of initial body weight) led to significant increases in both iso- and anteisoBCFAs (Table 2, Supplementary Table 1), so that total BCFA levels after surgery were no longer significantly different between groups (Fig. 1A). The OAGB-induced increase in circulating BCFA was similar in patients with morbid obesity with and without T2DM (Supplementary Fig. 1A, B).

Comparative analysis of FA profiles in patients with morbid obesity pre- and post-OAGB and in lean controls demonstrated many significant differences (Table 2). Both before and

Table 2 Serum fatty acids (\% of total fatty acid concentration) in patients with morbid obesity before and after surgery compared with lean controls. Values are mean $\pm \mathrm{SD}$. $L C$ lean controls, $O A G B$ one anastomosis gastric bypass, $B C F A s$ branched-chain saturated FAs, after surgery, patients with morbid obesity had lower OCFA levels than control subjects (Table 2). Moreover, preoperative serum levels of $n-3$ polyunsaturated FAs (PUFAs) and postoperative levels of both n-3 and n-6 PUFAs were significantly lower in subjects with morbid obesity than in lean subjects. On the other hand, both before and after OAGB, patients with morbid obesity had higher levels of monounsaturated FAs (MUFAs) than lean controls (Table 2).

\section{The Effect of OAGB on Serum BCAA Composition}

Since BCFAs can be synthetized from BCAA precursors in adipose tissue $[17,19]$, we measured pre- and post-OAGB concentrations of valine, leucine, and isoleucine in patients with morbid obesity and in lean controls. Total circulating BCAAs (leucine and isoleucine, but not valine) were

ECFAs even-chain saturated FAs, MUFAs monounsaturated FAs, OCFAs odd-chain saturated FAs, PUFAs polyunsaturated FAs, SFAs saturated FAs

\begin{tabular}{lcccccc}
\hline Fatty acid & Lean controls & Pre-OAGB & Post-OAGB & $p$ (pre-OAGB vs LC) & $p$ (pre- vs post-OAGB) & $p$ (post-OAGB vs LC) \\
\hline Total OCFAs & $0.649 \pm 0.111$ & $0.572 \pm 0.09$ & $0.581 \pm 0.01$ & 0.002 & 0.542 & 0.012 \\
Iso-BCFAs & $0.244 \pm 0.058$ & $0.190 \pm 0.05$ & $0.234 \pm 0.06$ & $<0.001$ & $<0.001$ & 0.437 \\
Anteiso-BCFAs & $0.167 \pm 0.046$ & $0.094 \pm 0.03$ & $0.135 \pm 0.05$ & $<0.001$ & $<0.001$ & 0.055 \\
Other BCFAs & $0.011 \pm 0.007$ & $0.010 \pm 0.004$ & $0.012 \pm 0.01$ & 0.493 & 0.390 & 0.005 \\
Total ECFAs & $32.1 \pm 1.87$ & $32.5 \pm 1.78$ & $32.8 \pm 2.40$ & 0.276 & 0.261 & 0.131 \\
Total SFAs & $35.3 \pm 1.84$ & $33.6 \pm 1.82$ & $33.9 \pm 2.60$ & 0.540 & 0.175 & 0.200 \\
Total MUFAs & $29.6 \pm 3.60$ & $33.6 \pm 2.64$ & $34.3 \pm 3.06$ & $<0.001$ & 0.115 & $<0.001$ \\
Total n-6 PUFAs & $34.1 \pm 3.70$ & $30.4 \pm 3.65$ & $29.5 \pm 4.13$ & $<0.001$ & 0.036 & 0.005 \\
Total n-3 PUFAs & $2.99 \pm 1.27$ & $2.44 \pm 0.69$ & $2.27 \pm 0.54$ & 0.032 & & $<$ \\
\hline
\end{tabular}


Fig. 1 Serum BCFA (A) and BCAA (B) levels in patients with morbid obesity before and after surgery compared with lean controls
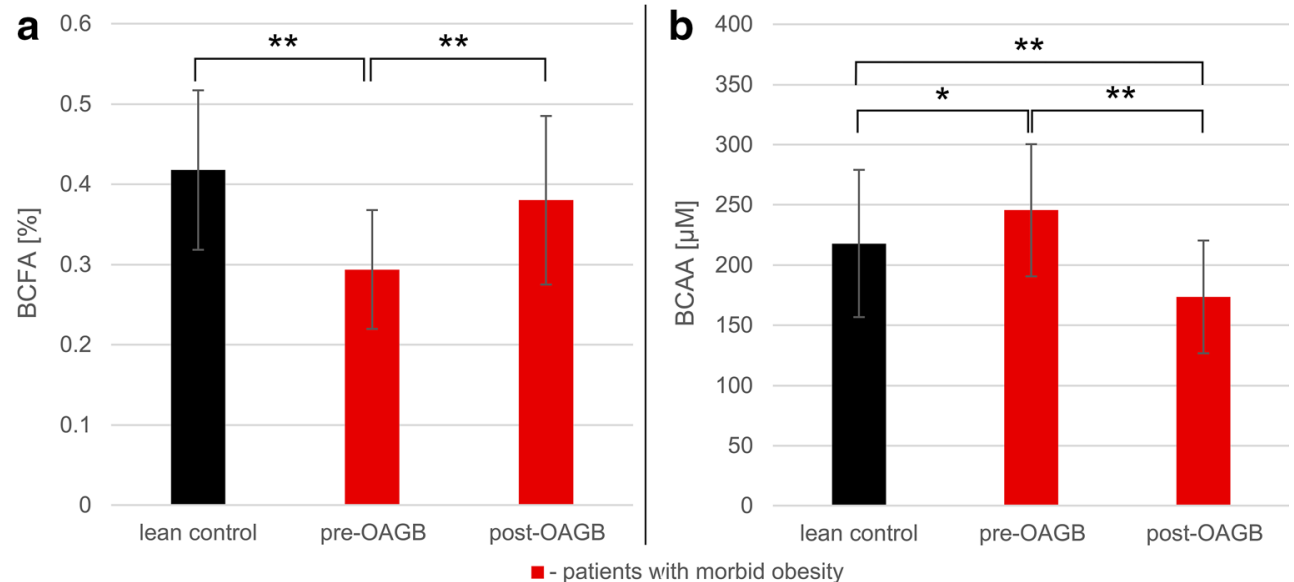

significantly greater in subjects with morbid obesity before surgery than in lean controls (Table 3 and Fig. 1B). OAGB significantly decreased the concentrations of all BCAAs, so that postoperative values were all significantly lower in subjects after bariatric surgery than in lean controls (Table 3 and Fig. 1B).

\section{Associations Between BCFAs and BCAAs with HOMA, Triglycerides, and BMI}

At baseline, we found that BCAAs correlated directly with the homeostasis model assessment (HOMA) index of insulin resistance $(r=0.30, p=0.007)$ and triglyceride concentration ( $r=0.33, p=0.003)$, whereas BCFAs correlated inversely with these two parameters (HOMA: $r=-0.28, p=0.012$; triglycerides: $r=-0.37, p=0.001)$. Furthermore, BMI correlated negatively with BCFA $(r=-0.60, p<0.001)$ and positively with BCAA $(r=0.27, p=0.015)$. Multiple linear regression analysis revealed that serum BCFA content was predicted most strongly by BMI $(p<0.001)$, whereas BCAA by triglycerides $(p=0.007)$.

\section{Expression of Genes Involved in BCAA Catabolism in Adipose Tissue}

We analyzed mRNA levels for the enzymes involved in BCAA catabolism in SAT and VAT. We did not find any statistically significant differences between subjects with morbid obesity and lean controls in the gene expression of $\mathrm{BCKDH}$ (isoforms A and B) and BCAT (isoforms 1 and 2) in SAT (BCKDHA $1.0 \pm 0.38$ vs $1.07 \pm 0.38, p=0.59$; $\mathrm{BCKDHB} 1.0 \pm 0.26$ vs $1.03 \pm 0.39, p=0.78$; BCAT1 $1.0 \pm$ 0.54 vs $1.18 \pm 0.66, p=0.22$; BCAT2 $1.0 \pm 0.35$ vs $0.86 \pm$ $0.22, p=0.11$; in control subjects and patients with obesity, respectively). However, the mRNA levels for $\mathrm{BCKDH}$ isoform B and both BCAT isoforms in VAT were significantly lower in subjects with morbid obesity than in lean controls (Fig. 2A). Accordingly, the lower gene expression of enzymes catabolizing BCAAs (which are involved in the synthesis of BCFAs) was accompanied by lower BCFA levels in VAT in subjects with morbid obesity than in lean subjects (Fig. 2B). In SAT, the levels of BCFA did not differ between control subjects and patients with obesity $(0.44 \pm 0.15$ vs $0.47 \pm 0.11, p=$ 0.34 , respectively).

\section{Discussion}

\section{Association Between Serum Levels of BCFA and BCAA in Obese Subjects}

In mammalian tissues, BCFAs rarely constitute more than 1$2 \%$ of the total FA pool, and the results from previous studies imply that they are primarily synthesized by intestinal bacteria, or consumed with dairy products or ruminant meat [27, 28]. However, recent evidence suggests that BCFAs may also

Table 3 Serum branched-chain amino acids $(\mu \mathrm{mol} / \mathrm{l})$ in patients with morbid obesity before and after surgery compared with lean controls. Values are mean $\pm \mathrm{SD}$. $L C$ lean controls, $O A G B$ one anastomosis gastric bypass

\begin{tabular}{lcrlccc}
\hline & Lean controls & Pre-OAGB & $\begin{array}{l}\text { Post- } \\
\text { OAGB }\end{array}$ & $p$ (pre-OAGB vs LC) & $p$ (pre- vs post-OAGB) & $p$ (post-OAGB vs LC) \\
\hline Valine & $128 \pm 35.8$ & $123 \pm 25.9$ & $97.7 \pm 24.5$ & 0.42 & $<0.001$ & $<0.001$ \\
Leucine & $57.6 \pm 19.2$ & $77.1 \pm 20.0$ & $47.4 \pm 18.3$ & $<0.001$ & $<0.001$ & 0.02 \\
Isoleucine & $32.0 \pm 12.7$ & $45.9 \pm 14.5$ & $28.4 \pm 10.5$ & $<0.001$ & $<0.001$ & 0.19 \\
\hline
\end{tabular}


Fig. 2 mRNA levels for BCKDHA, BCKDHB, BCAT1, and BCAT2 in VAT of patients with morbid obesity compared with lean controls (A). BCFA levels in VAT of patients with morbid obesity compared with lean controls (B)

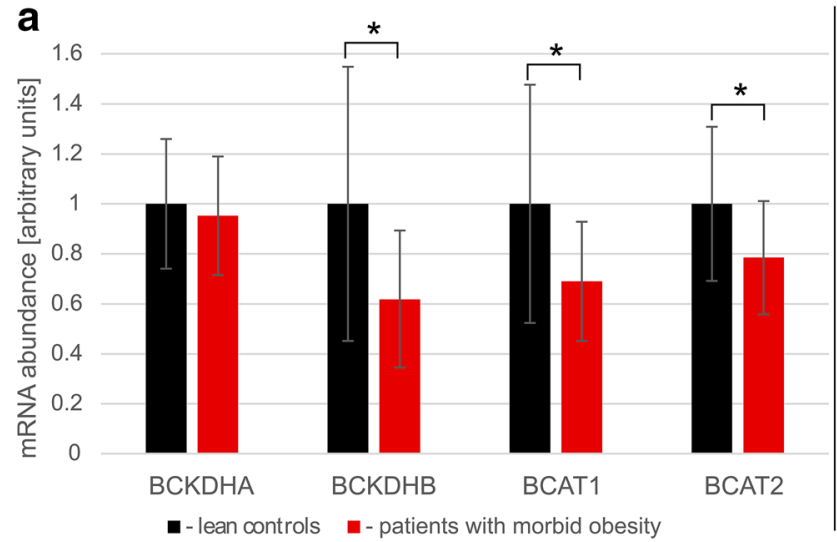

be synthesized in mammalian adipose tissue from BCAAderived precursors [17]. Despite their very low circulating levels, BCFAs have been associated with several beneficial metabolic outcomes in human subjects [16]. Recently, we demonstrated that morbid obesity is associated with decreased serum iso-BCFA levels. Our present study confirms this finding and further demonstrates that bariatric surgery-induced weight loss increases serum BCFAs. This may constitute another beneficial metabolic effect of OAGB in patients with morbid obesity. The results of our previous study suggest that BCFA levels in these patients are likely independent of their dietary habits [16]. Thus, the lower levels of BCFAs in people with morbid obesity and the increase in circulating BCFA after surgery may be associated with alterations in the metabolism of BCAAs, namely valine, leucine, and isoleucine, which can serve as precursors of BCFAs [17, 19]. In line with this hypothesis, the first step in the formation of BCFA primers is the conversion of BCAAs to $\alpha$-keto acids by mitochondrial and cytosolic isoforms of BCAT, followed by production of primer-CoA esters in a reaction catalyzed by the $\mathrm{BCKDH}$ complex [29]. While the reaction catalyzed by mitochondrial BCKDH is the rate-limiting step in the synthesis of BCFAs from BCAAs [18], in some tissues (e.g., liver), its products can also be transformed into intermediates of the Krebs cycle. During the last step, the primer-CoA esters derived from BCAAs are elongated into BCFAs [30].

Amino acids comprise a group of metabolites that undergoes significant alterations after bariatric surgery. Many previous studies demonstrated that another type of bypass procedure, Roux-en-Y gastric bypass (RYGB), led to significant decreases in serum BCAA levels [9, 31-33]. Importantly, the effect of RYGB on serum BCAA concentration persisted regardless of the time elapsed since surgery [34]. According to Wijayatunga et al. [9], the RYGB-induced decrease in BCAA may be a consequence of enhanced BCAA catabolism or reduced protein intake after surgery [35]. Our present study confirms these observations after another type of intestinal bypass (OAGB). To the best of our knowledge, ours is the first study to demonstrate the effect of OAGB on serum BCAAs. Previous studies showed that high concentrations of leucine, isoleucine, and valine are associated with diabetes mellitus, insulin resistance, and coronary artery disease [28]. For example, Newgard et al. [20] reported that persons with morbid obesity had higher BCAA levels than lean controls, which contributed to the development of insulin resistance and diabetes. In view of these findings, the post-OAGB decrease in BCAA concentration could be considered another beneficial effect of bariatric surgery for obesity, even though the precise mechanism responsible remains unknown, and cannot be elucidated by our study. Considering that obesity and bariatric surgery had diametrically opposite effects on serum BCAAs and BCFAs (Fig. 1A and B), it is not unreasonable to hypothesize that the lower levels of BCFAs in subjects with morbid obesity may be associated with slower transformation of BCAAs into BCFAs. In line with this hypothesis, the OAGB-induced changes in serum levels of these metabolites may be explained by an improvement of BCAA to BCFA transformation. However, according to Yao et al. [36], the increase in BCAA levels in obesity likely results from an inability to adequately suppress proteolysis rather than from a decrease in the catabolism of BCAA. Thus, the mechanisms for the decrease in BCAA levels after surgeryinduced weight loss remain elusive and should be assessed in future studies.

\section{Relationship Between BCAA Catabolism and BCFA Synthesis in Adipose Tissue of Obese Patients}

As BCFAs can be produced during BCAA catabolism in adipose tissue [17, 19], we analyzed the expression of genes encoding $\mathrm{BCKDH}$ and $\mathrm{BCAT}$, i.e., the enzymes responsible for conversion of BCAAs to BCFAs, in VAT and SAT of patients with morbid obesity and lean controls. We detected no significant differences between groups in SAT, but patients with morbid obesity had significantly lower mRNA levels than lean subjects for BCKDHB, BCAT1, and BCAT2 in 
VAT. Accordingly, this was associated with lower BCFA level in VAT in subjects with morbid obesity than in lean subjects, which may represent the functional endpoint of the downregulation of the aforementioned genes. Our findings are consistent with the results published by Boulet et al. [21] who observed that BCAT2, BCKDHA, and BCKDHB mRNA and protein levels in VAT, but not SAT, of patients with morbid obesity were significantly lower than in lean controls. Also, Serralde-Zúniga et al. [37] reported that gene expressions of BCKDH E1 $\alpha$ and BCAT2 in omental adipose tissue (a VAT depot) of persons with morbid obesity with insulin resistance were significantly lower than in overweight persons without insulin resistance. Collectively, these observations suggest that a decrease in serum BCFAs and a concomitant increase in serum BCAAs in patients with morbid obesity might be a consequence of attenuated conversion of BCAAs into BCFAs in VAT. Unfortunately, we were not able to obtain adipose tissue samples from patients after surgery to verify if the increase in serum BCFAs and the decrease in serum OCFAs were associated with a post-OAGB upregulation of enzymes involved in BCAA catabolism in VAT. However, She et al. [35] reported a significant upregulation of BCAT and a nonsignificant upregulation of BCKDH in VAT (and SAT) of patients with morbid obesity after gastric bypass. Furthermore, Su et al. [17] reported increased SAT BCFA levels and higher expression of fatty acid synthase gene in patients with morbid obesity 1 year after RYGB. Based on those findings, synthesis of BCFAs in adipose tissue likely depends on both BCAA catabolism and FAS-catalyzed lipogenesis [17].

\section{Study Limitations}

Our study has several limitations. First, we were not able to obtain adipose tissue samples from patients after surgery to assess its effect on the gene expression of enzymes involved in BCAA catabolism. Second, we assessed metabolic function on the basis of static measurements of cardiovascular risk markers in the fasting state and did not employ methodologies to assess dynamic changes in metabolism (e.g., glucose tolerance tests or glucose/insulin clamps). Third, our cohort was relatively small, but even with this sample size, the statistical significance of the results was quite robust and therefore convincing. The major strength of our study is that, for the first time, we simultaneously determined the effect of bariatric surgery on circulating levels of BCAA and BCFA.

\section{Conclusion}

In this study, we showed that OAGB-induced weight loss resulted in significant increases in serum BCFAs and significant decreases in serum BCAAs in patients with morbid obesity. Moreover, our findings suggest that serum levels of BCFAs and BCAAs may be influenced by the relationship between BCAA catabolism and BCFA synthesis in VAT of subjects with morbid obesity. We speculate that the postOAGB changes in BCFA and BCAA levels may be associated with some metabolic benefits, such as improved glucose homeostasis, but the underlying mechanisms remain to be elucidated.

Funding Information This study was supported by the Medical University of Gdansk (grant nos. ST40, ST89) and by the National Science Centre of Poland (grant no. NCN 2016/21/D/NZ5/00219).

\section{Compliance with Ethical Standards}

Conflict of Interest The authors declare that they have no conflict of interest.

Ethical Approval All procedures performed in the studies involving human participants were in accordance with the ethical standards of the institutional and/or national research committee and with the 1964 Helsinki declaration and its later amendments or comparable ethical standards.

Informed Consent Informed consent was obtained from all participants included in the study before any study-related procedures take place.

Open Access This article is distributed under the terms of the Creative Commons Attribution 4.0 International License (http:// creativecommons.org/licenses/by/4.0/), which permits unrestricted use, distribution, and reproduction in any medium, provided you give appropriate credit to the original author(s) and the source, provide a link to the Creative Commons license, and indicate if changes were made.

\section{References}

1. Athyros VG, Tziomalos K, Karagiannis A, et al. Cardiovascular benefits of bariatric surgery in morbidly obese patients. Obes Rev. 2011;12:515-24.

2. Wylezoł M, Pasnik K, Dabrowiecki S, et al. Polish recommendations for bariatric surgery. Wideochir Inne Tech Maloinwazyjne. 2009;4(Suppl 1):S5-8.

3. Ramos-Molina B, Castellano-Castillo D, Alcaide-Torres J, et al. Differential effects of restrictive and malabsorptive bariatric surgery procedures on the serum lipidome in obese subjects. J Clin Lipidol. 2018;12:1502-12.

4. Mika A, Kaska L, Proczko-Stepaniak M, et al. Evidence that the length of bile loop determines serum bile acid concentration and glycemic control after bariatric surgery. Obes Surg. 2018;28:340514.

5. Lee W-J, Yu P-J, Wang W, et al. Laparoscopic Roux-en-Y versus mini-gastric bypass for the treatment of morbid obesity. Ann Surg. 2005;242:20-8.

6. Rutledge R. The mini-gastric bypass: experience with the first 1, 274 cases. Obes Surg. 2001;11:276-80.

7. Robert M, Espalieu P, Pelascini E, et al. Efficacy and safety of one anastomosis gastric bypass versus Roux-en-Y gastric bypass for obesity (YOMEGA): a multicentre, randomised, open-label, noninferiority trial. Lancet. 2019;393:1299-309. 
8. Courcoulas AP, Yanovski SZ, Bonds D, et al. Long-term outcomes of bariatric surgery: a National Institutes of Health symposium. JAMA Surg. 2014;149:1323-9.

9. Wijayatunga NN, Sams VG, Dawson JA, et al. Roux-en-Y gastric bypass surgery alters serum metabolites and fatty acids in patients with morbid obesity. Diabetes Metab Res Rev. 2018;8:e3045.

10. Rezanka T, Sigler K. Odd-numbered very-long-chain fatty acids from the microbial, animal and plant kingdoms. Prog Lipid Res. 2009;48:206-38

11. Wongtangtintharn $\mathrm{S}, \mathrm{Oku} \mathrm{H}$, Iwasaki $\mathrm{H}$, et al. Incorporation of branched-chain fatty acid into cellular lipids and caspaseindependent apoptosis in human breast cancer cell line, SKBR-3. Lipids Health Dis. 2005;4:29.

12. Vlaeminck B, Fievez V, Cabrita ARJ, et al. Factors affecting oddand branched-chain fatty acids in milk: a review. Anim Feed Sci Technol. 2006;131:389-417.

13. Yan $Y$, Wang $Z$, Wang $X$, et al. Branched chain fatty acids positional distribution in human milk fat and common human food fats and uptake in human intestinal cells. J Funct Foods. 2017;29:172-7.

14. Warensjo E, Jansson J-H, Berglund L, et al. Estimated intake of milk fat is negatively associated with cardiovascular risk factors and does not increase the risk of a first acute myocardial infarction. A prospective case-control study. Br J Nutr. 2004;91:635-42.

15. Ran-Ressler RR, Bae $S$, Lawrence $P$, et al. Branched-chain fatty acid content of foods and estimated intake in the USA. Br J Nutr. 2014;112:565-72.

16. Mika A, Stepnowski P, Kaska L, et al. A comprehensive study of serum odd- and branched-chain fatty acids in patients with excess weight. Obesity. 2016;24:1669-76.

17. Su X, Magkos F, Zhou D, et al. Adipose tissue monomethyl branched-chain fatty acids and insulin sensitivity: effects of obesity and weight loss. Obesity. 2015;23:329-34.

18. Crown SB, Marze N, Antoniewicz MR. Catabolism of branched chain amino acids contributes significantly to synthesis of oddchain and even-chain fatty acids in 3T3-L1 adipocytes. Tochtrop GP, editor. PLoS One. 2015;10:e0145850.

19. Wallace M, Green CR, Roberts LS, et al. Enzyme promiscuity drives branched-chain fatty acid synthesis in adipose tissues. Nat Chem Biol. 2018;14:1021-31.

20. Newgard CB, An J, Bain JR, et al. A branched-chain amino acidrelated metabolic signature that differentiates obese and lean humans and contributes to insulin resistance. Cell Metab. 2009;9: 311-26.

21. Boulet MM, Chevrier G, Grenier-Larouche T, et al. Alterations of plasma metabolite profiles related to adipose tissue distribution and cardiometabolic risk. Am J Physiol Endocrinol Metab. 2015;309: E736-46.

22. Fried M, Yumuk V, Oppert J-M, et al. Interdisciplinary European guidelines on metabolic and bariatric surgery. Obes Facts. 2013;6: 449-68.

23. García-Caballero M, Carbajo M. One anastomosis gastric bypass: a simple, safe and efficient surgical procedure for treating morbid obesity. Nutr Hosp. 2004;19:372-5.
24. Folch J, Lees M, Sloane Stanley GH. A simple method for the isolation and purification of total lipides from animal tissues. $\mathrm{J}$ Biol Chem. 1957;226:497-509.

25. Olkowicz M, Debski J, Jablonska P, et al. Application of a new procedure for liquid chromatography/mass spectrometry profiling of plasma amino acid-related metabolites and untargeted shotgun proteomics to identify mechanisms and biomarkers of calcific aortic stenosis. J Chromatogr A. 2017;1517:66-78.

26. Piehler AP, Grimholt RM, Ovstebo R, et al. Gene expression results in lipopolysaccharide-stimulated monocytes depend significantly on the choice of reference genes. BMC Immunol. 2010;11:21.

27. Adamska A, Rutkowska J. Odd- and branched-chain fatty acids in milk fat - characteristic and health properties. Postepy Hig Med Dosw. 2014;68:957-66.

28. Newgard CB. Interplay between lipids and branched-chain amino acids in development of insulin resistance. Cell Metab. 2012;15: 606-14.

29. She P, Olson KC, Kadota Y, et al. Leucine and protein metabolism in obese Zucker rats. PLoS One. 2013;8:e59443.

30. Christie WW, Han X. Lipid analysis : isolation, separation, identification and lipidomic analysis. Oily Press, an imprint of PJ Barnes \& Associates; 2010.

31. Samczuk P, Ciborowski M, Kretowski A. Application of metabolomics to study effects of bariatric surgery. J Diabetes Res. 2018;2018:1-13.

32. Laferrere B, Reilly D, Arias S, et al. Differential metabolic impact of gastric bypass surgery versus dietary intervention in obese diabetic subjects despite identical weight loss. Sci Transl Med. 2011;3: 80re2.

33. Arora T, Velagapudi V, Pournaras DJ, et al. Roux-en-Y gastric bypass surgery induces early plasma metabolomic and lipidomic alterations in humans associated with diabetes remission. PLoS One. 2015;10:e126401.

34. Lopes TIB, Geloneze B, Pareja JC, et al. Blood metabolome changes before and after bariatric surgery: a ${ }^{1} \mathrm{H}$ NMR-based clinical investigation. Omics. 2015;19:318-27.

35. She P, Van Horn C, Reid T, et al. Obesity-related elevations in plasma leucine are associated with alterations in enzymes involved in branched-chain amino acid metabolism. Am J Physiol Metab. 2007;293:E1552-63.

36. Yao J, Kovalik JP, Lai OF, et al. Comprehensive assessment of the effects of sleeve gastrectomy on glucose, lipid, and amino acid metabolism in Asian individuals with morbid obesity. Obes Surg. 2018;29:149-58.

37. Serralde-Zuniga AE, Guevara-Cruz M, Tovar AR, et al. Omental adipose tissue gene expression, gene variants, branched-chain amino acids, and their relationship with metabolic syndrome and insulin resistance in humans. Genes Nutr. 2014;9:1-10.

Publisher's Note Springer Nature remains neutral with regard to jurisdictional claims in published maps and institutional affiliations. 


\section{Affiliations}

Alicja Pakiet ${ }^{1}$ - Maciej Wilczynski ${ }^{2}$ - Olga Rostkowska ${ }^{2}$. Justyna Korczynska ${ }^{3} \cdot$ Patrycja Jabłonska $^{4} \cdot$ Lukasz Kaska $^{2}$. Monika Proczko-Stepaniak ${ }^{2}$ - Ewa Sobczak ${ }^{5,6} \cdot$ Piotr Stepnowski $^{1} \cdot$ Faidon Magkos $^{7} \cdot$ Tomasz Sledzinski $^{3}$ (D) Adriana Mika ${ }^{1,3}$

1 Department of Environmental Analytics, Faculty of Chemistry, University of Gdansk, Wita Stwosza 63, 80-308 Gdansk, Poland

2 Department of General, Endocrine and Transplant Surgery, Faculty of Medicine, Medical University of Gdansk, 17 Smoluchowskiego, 80-214 Gdansk, Poland

3 Department of Pharmaceutical Biochemistry, Faculty of Pharmacy, Medical University of Gdansk, 1 Debinki Str., 80-

211 Gdansk, Poland

4 Department of Biochemistry, Faculty of Medicine, Medical

University of Gdansk, Debinki 1, 80-211 Gdansk, Poland
5 Department of Clinical Nutrition, Faculty of Health Sciences, Medical University of Gdansk, Debinki 7, 80-211 Gdansk, Poland

6 Western Sussex Hospitals NHS Foundation Trust, Worthing Hospital, Worthing, Lyndhurst Rd, Worthing, West Sussex BN11 2DH, UK

7 Department of Nutrition, Exercise and Sports, Faculty of Science, University of Copenhagen, Bülowsvej 17, 1870 Frederiksberg C, Denmark 